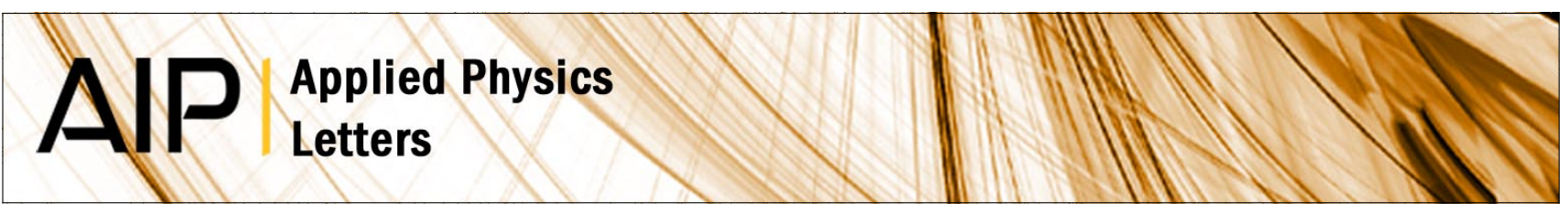

\title{
Electrical conductivity relaxation in thin-film yttria-stabilized zirconia
}

A. Rivera, J. Santamaría, and C. León

Citation: Appl. Phys. Lett. 78, 610 (2001); doi: 10.1063/1.1343852

View online: http://dx.doi.org/10.1063/1.1343852

View Table of Contents: http://apl.aip.org/resource/1/APPLAB/v78/i5

Published by the American Institute of Physics.

Additional information on Appl. Phys. Lett.

Journal Homepage: http://apl.aip.org/

Journal Information: http://apl.aip.org/about/about_the_journal

Top downloads: http://apl.aip.org/features/most_downloaded

Information for Authors: http://apl.aip.org/authors

\section{ADVERTISEMENT}

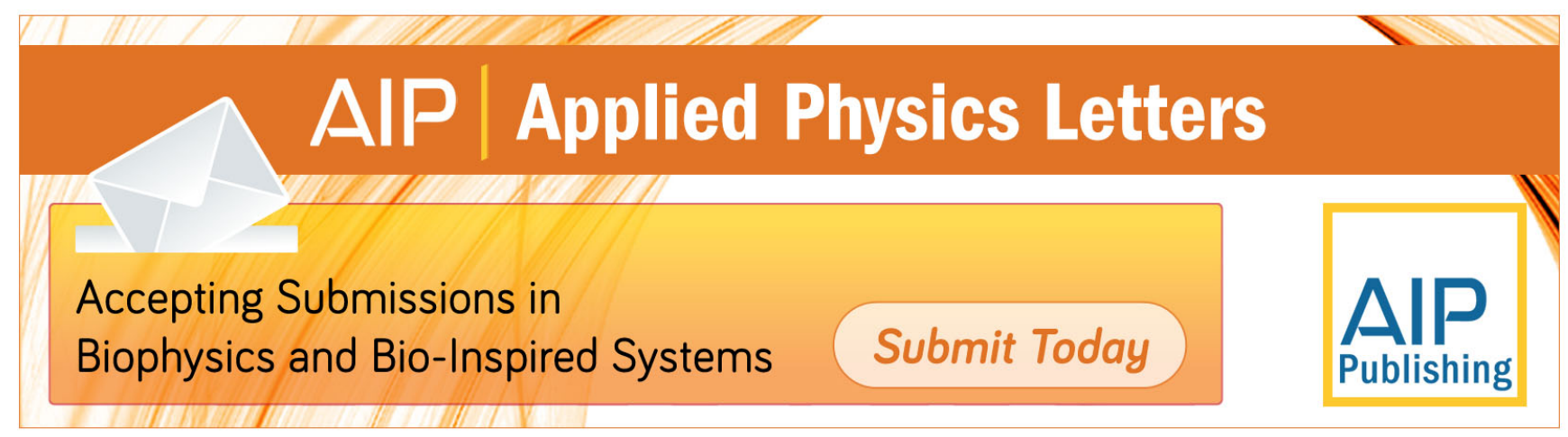




\title{
Electrical conductivity relaxation in thin-film yttria-stabilized zirconia
}

\author{
A. Rivera, J. Santamaría, and C. León ${ }^{\text {a) }}$ \\ GFMC, Departamento de Física Aplicada III, Facultad de Ciencias Físicas, Universidad Complutense \\ de Madrid, Avda. Complutense s/n, 28040 Madrid, Spain
}

(Received 9 August 2000; accepted for publication 27 November 2000)

\begin{abstract}
We report on complex admittance measurements on $\mathrm{ZrO}_{2}: \mathrm{Y}_{2} \mathrm{O}_{3}$ (YSZ) thin films in the parallel plate geometry. Highly textured YSZ thin films, grown by rf sputtering, allow measuring complex admittance free of the effect of charge blocking at grain boundaries. We have examined low-temperature (close to room temperature) regime dominated by association of oxygen vacancies. Complex admittance analyzed in terms of the modulus formalism supplies information on correlation effects in ion motion and allows obtaining an association energy for the oxygen vacancies of $0.45 \mathrm{eV}$, in agreement with previous theoretical calculations. (C) 2001 American Institute of Physics. [DOI: 10.1063/1.1343852]
\end{abstract}

Thin films of ionically conducting materials have opened new paths for applications in thin film batteries and other miniaturized devices like fuel cells or gas sensors, and have focused considerable attention in recent years. ${ }^{1-3}$ Yttria stabilized zirconia, $\mathrm{ZrO}_{2}: \mathrm{Y}_{2} \mathrm{O}_{3}$, (YSZ) is one of the most extensively studied fast anionic conductors due to the high values of the oxygen conductivity $(0.1 \mathrm{~S} / \mathrm{cm})$ at high temperatures $\left(1000{ }^{\circ} \mathrm{C}\right) .{ }^{4}$ Doping with $\mathrm{Y}_{2} \mathrm{O}_{3}$ is known to stabilize at room temperature the cubic fluorite structure of $\mathrm{ZrO}_{2}$, and to supply the oxygen vacancies responsible for the ionic conduction. Although the high-temperature conductivity of bulk YSZ samples has been extensively investigated, ${ }^{5-7}$ open questions remain on the low temperature (close to room temperature) conduction mechanism. Oxygen vacancies are known to be associated to defect centers, and since not all oxygen sites are equivalent, this may enhance correlations in the low temperature diffussion process. ${ }^{7,8}$ Aside from their interest for applications, thin film samples are very adequate to address this issue for two main reasons. Most electrical conductivity measurements in solid electrolytes require parallel plate geometry, such that the contacts surface (S) is large compared to the sample thickness (d). Sometimes this may not be easy to achieve in bulk samples; thin films, on the other hand, are naturally well suited to match this requirement, and large $\sqrt{S} / d$ ratios allow extending the measurable conductivity values in the low temperature range. Additionally, ceramic bulk samples show significant blocking of charge carriers at grain boundaries, which frequently obscures the bulk response making the determination of the conductivity somehow difficult. In this respect, highly textured (or epitaxial) films in parallel plate geometry constitute an interesting alternative to measure the bulk ionic conductivity.

In this letter, we report a study of the electrical conductivity relaxation in thin film YSZ samples using the perpendicular (parallel plate) geometry. This has allowed examining the relatively unexplored low temperature regime dominated by association of oxygen vacancies. Complex admittance analyzed in terms of the modulus formalism sup-

${ }^{a)}$ Electronic mail: carletas@eucmax.sim.ucm.es plies information on correlation effects in ion motion and allows obtaining an association energy for the oxygen vacancies in agreement with previous theoretical calculations.

YSZ films were deposited by rf diode sputtering on $\mathrm{MgO}(100)$ single crystal substrates. Prior to YSZ deposition, a gold layer $200 \AA$ thick was evaporated on the substrates to act as a bottom contact for electrical characterization. Growth temperature was $650{ }^{\circ} \mathrm{C}$ and the sputtering atmosphere was an $\mathrm{Ar} / \mathrm{O}_{2}$ admixture. Sandwich structures for electrical measurements were defined evaporating $1 \mathrm{~mm}^{2}$ gold spots on the surface of films. Film thickness was in the range $500-800 \mathrm{~nm}$ to avoid shorts through pinholes. Admittance spectroscopy was measured in the frequency range 20 $\mathrm{Hz}-1 \mathrm{MHz}$ using an LCR meter HP 4284A, at temperatures comprised between 300 and $580 \mathrm{~K}$. Measurements were conducted under high purity $\mathrm{N}_{2}$ flow to ensure an inert atmosphere.

Thin films had an yttria molar content of $8 \%$, i.e., $8 \% \mathrm{~mol} \mathrm{Y}_{2} \mathrm{O}_{3}-\mathrm{ZrO}_{2}$, determined by EDS from the $\mathrm{Y} / \mathrm{Zr}$ ratio and using an YSZ single crystal as a standard. The inset of Fig. 1 shows an $\mathrm{x}$-ray diffraction pattern of a typical YSZ film grown on $\mathrm{MgO} / \mathrm{Au}$. Films are strongly $(h 00)$ textured despite the presence of the $200 \AA$ thick base gold layer. Full width at half maximum (FWHM) of the rocking curves around the (200) peak are found to be $0.7^{\circ}$ wide. Cross sectional SEM observations of YSZ films confirmed a column like structure with no grain boundaries perpendicular to the growth direction. The highly textured growth of the samples ensures that in the parallel plate geometry current flow is not limited by grain boundaries.

Figure 1 shows a complex specific impedance plot of the thin film sample at a temperature of $558 \mathrm{~K}$. For comparison, data of a sintered ceramic at a temperature of $572 \mathrm{~K}$ and a single crystal at $558 \mathrm{~K}$ have been included in the same figure. Since the Y/Zr ratio were slightly different for the various samples, temperatures have been chosen for all samples to have a similar value of the dc conductivity. It can be readily observed that a second semicircle appears in the ceramic sample as a result of charge blocking at grain boundaries, while for the thin film sample one can clearly separate the bulk contribution (of interest here) from other relaxation 


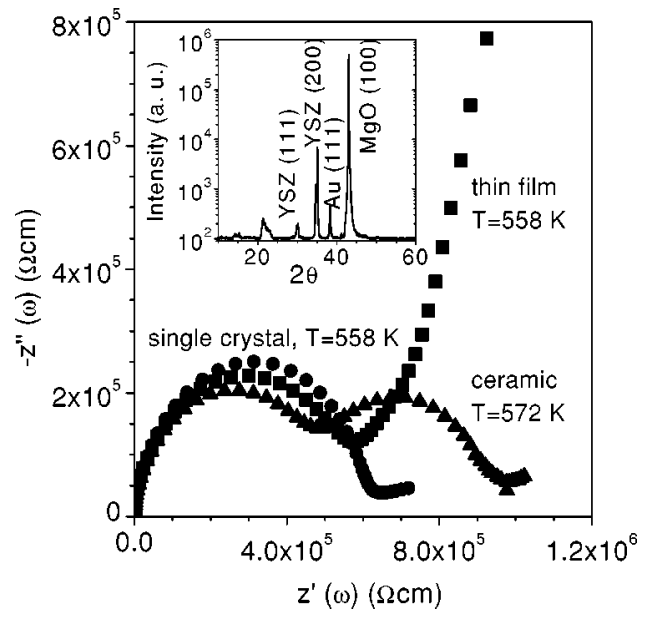

FIG. 1. Complex specific impedance plots for thin film, ceramic, and syngle cryslal YSZ samples of similar yttria contents. Temperatures have been selected to yield a similar dc conductivity value for all samples. The inset shows an X-ray diffraction pattern of a YSZ thin film grown on $\mathrm{MgO}$ with a 200 Å gold base layer.

processes including grain boundary blocking effects. Figure 2(a) shows the real part of the conductivity as a function of frequency for several temperatures. Only at the highest temperatures a decrease of the conductivity is observed at the lowest frequencies, which may be ascribed to blocking of carriers at the electrodes. Aside from this effect, a well developed nondispersive dc conductivity is observed over a wide temperature range with no signs of charge blocking at grain boundaries, as expected from the columnar growth. When frequency is increased a crossover occurs to a power law dependent ac conductivity of the form $\omega^{n}$. Complex conductivity can thus be phenomenologically described by the Jonscher's expression: ${ }^{9}$

$$
\sigma^{*}(\omega)=\sigma_{\mathrm{dc}}\left[1+\left(j \omega / \omega_{p}\right)^{n}\right],
$$

frequently observed in ionic conductors. The exponent $n$ was close to $0.6, \omega_{p}$ is a crossover frequency, and $\sigma_{\mathrm{dc}}$ is the dc conductivity. Power laws in the conductivity have been ascribed to a nonrandom hopping mechanism resulting from many body interactions between charge carriers and the fractional exponent $n$ is a measure of the index of correlation. ${ }^{9,10}$ When temperature is decreased an increase in $n$ is observed towards $n=1$. This regime, known as "constant loss," has been previously observed in other materials, ${ }^{11}$ and although its origin is still not well understood, it seems to be related to vibration of ions within their wells. ${ }^{12}$
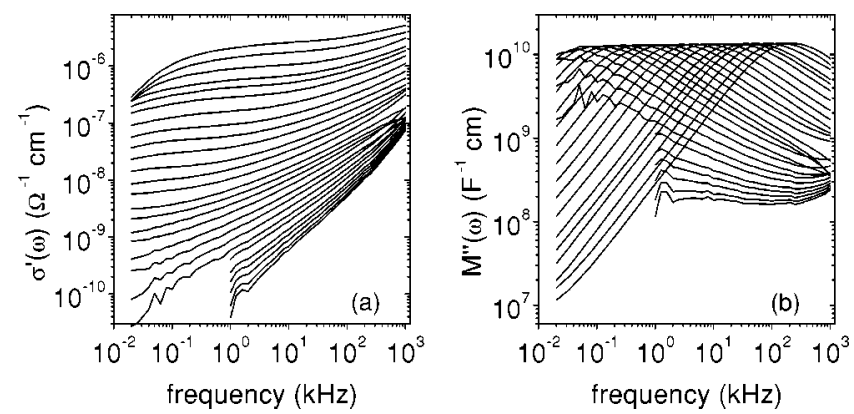

FIG. 2. Frequency dependence of the real part of the conductivity (a) and of the imaginary part of the electric modulus (b) at several temperatures between 307 and $572 \mathrm{~K}$.

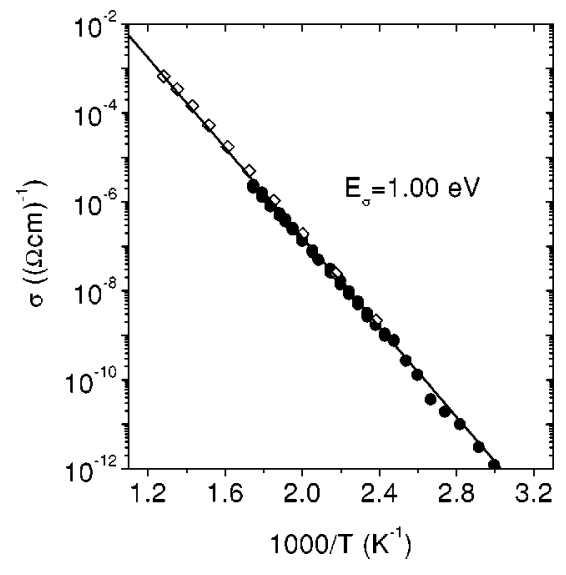

FIG. 3. Temperature dependence of dc conductivity in thin film YSZ ( $8 \% \mathrm{~mol} \mathrm{Y}_{2} \mathrm{O}_{3}$ ) (solid circles). Data correspond to a bulk sample with similar composition are shown for comparison (open diamonds). Solid line is a fit to an Arrhenius temperature dependence.

An alternative time domain image of the effects of correlations in the relaxation of the conductivity can be obtained from the modulus formalism, ${ }^{13,14}$ which describes the relaxation of the electric field $\mathbf{E}$ at constant displacement vector D. Figure 2(b) shows the imaginary part of the electric modulus at several temperatures. The electric field decay function $\Phi(t)$ is obtained as:

$$
M^{*}(\omega)=M_{\infty}\left[1-\int_{0}^{\infty}(-d \Phi / d t) e^{-j \omega t} d t\right]
$$

where $\mathrm{M}_{\infty}$ is the inverse of the high frequency dielectric permittivity. Kohlrausch-Williams-Watts (KWW), "stretched" exponential decay functions of the form:

$$
\Phi(t)=\exp \left[-\left(t / \tau^{*}\right)^{\beta}\right]
$$

have been obtained fitting modulus plots to expression (2). $\tau^{*}$ is a temperature activated relaxation time and $0<\beta<1$. The stretched exponential parameter $\beta$ describes the slowing down of the relaxation process as a result of correlated ion hopping, ${ }^{15-17}$ such that one expects $\beta$ to be close to zero for strongly correlated ion motion and one for random Debyelike hops. An exponent $\beta=0.45 \pm 0.02$ is obtained from the fits over the whole temperature range. Following the method proposed by other authors ${ }^{15}$ an average relaxation time can be obtained as

$$
\langle\tau\rangle=\int_{0}^{\infty} \Phi(t) d t=\Gamma(1 / \beta) \tau^{*} / \beta,
$$

where $\Gamma$ is the Euler's gamma function. The dc conductivity can thus be obtained according to the expression ${ }^{15-17} \sigma_{0}$ $=\varepsilon_{0} \varepsilon_{\infty} /\langle\tau\rangle$, where $\varepsilon_{0}$ is the free space dielectric constant and $\varepsilon_{\infty}$ is the high frequency relative permittivity, which takes a value of 27 , practically temperature independent. The fact that modulus maxima of Fig. 2(b) have a temperature independent amplitude supports the temperature independence of $\beta$ and $\varepsilon_{\infty}$.

Figure 3 displays the dc conductivity data, obtained from fits to expressions (1) and (2), in an Arrhenius plot. An activation energy $E_{\sigma}=1.00 \pm 0.03 \mathrm{eV}$ is obtained over more than nine decades. This activation energy agrees with previously reported values for single crystalline or ceramic 
samples with similar compositions. ${ }^{18,19}$ For comparison, data corresponding to a bulk sample of the same composition have been included in the figure. It can be observed that the use of thin film samples allows extending the measuring range.

According to Ngai's coupling mode ${ }^{20}$ the dc conductivity activation energy and the exponent $\beta$ can be used to obtain the single ion microscopic activation energy, $E_{m}$, as $E_{m}=\beta E_{\sigma}$. A value of $0.45 \mathrm{eV}$ results for $E_{m}$, which is in very good agreement with the value of $0.49 \mathrm{eV}^{\text {reported }}{ }^{21}$ for the association energy of oxygen vacancies. This strongly suggests that the microscopic activation may be triggered by oxygen vacancy association, and that the lattice (as opposed to ion-ion interaction) may be playing a significant role in establishing correlations for ion motion.

In summary, we have grown highly textured YSZ thin films free of grain boundaries parallel to the substrate. Complex admittance measurements in the perpendicular geometry have allowed to extend the low temperature conductivity measurements down to the $10^{-12} \Omega^{-1} \mathrm{~cm}^{-1}$ range to study the effect of correlations on ion transport. The analysis of the dispersive admittance using the modulus formalism supplies a temperature independent $\mathrm{KWW}$ exponent of 0.45 . Using the coupling model, a microscopic activation energy of 0.45 $\mathrm{eV}$ is obtained, which agrees with previous calculations of the association energy of oxygen vacancies.
${ }^{1}$ Proceedings of the Tenth International Conference on Solid State Ionics, Special Volume of Solid State Ionics, edited by B. V. R. Chowdari (North-Holland, Amsterdam, 1996).

${ }^{2}$ Proceedings of the Fifth International Symposium on Solid Oxide Fuel Cells (Electrochemical Society Pennington, NJ, 1997).

${ }^{3}$ K. Suzuki, M. Kubo, Y. Oumi, R. Miura, H. Takaba, A. Fahmi, A. Chatterjee, K. Teraishi, and A. Miyamoto, Appl. Phys. Lett. 73, 1502 (1998).

${ }^{4}$ See the review by T. H. Etsell and S. N. Flengas, Chem. Rev. 70, 339 (1970).

${ }^{5}$ J. M. Dixon, L. D. La Grange, U. Merten, C. F. Miller, and J. T. Porter, J. Electrochem. Soc. 110, 276 (1963).

${ }^{6}$ D. W. Stickler and W. G. Carlson, J. Am. Ceram. Soc. 47, 122 (1964).

${ }^{7}$ J. D. Solier, I. Cachadiña, and A. Dominguez-Rodriguez, Phys. Rev. B 48, 3704 (1993).

${ }^{8}$ J. P. Goff, W. Hayes, S. Hull, M. T. Hutchings, and K. N. Clausen, Phys. Rev. B 59, 14202 (1999).

${ }^{9}$ A. K. Jonscher, in Dielectric Relaxation in Solids (Chelsea Dielectric, London, 1983).

${ }^{10}$ K. Funke, Prog. Solid State Chem. 22, 111 (1993).

${ }^{11}$ W. K. Lee, J. F. Liu, and A. S. Nowick, Phys. Rev. Lett. 67, 1559 (1991).

${ }^{12}$ K. L. Ngai, J. Chem. Phys. 110, 10576 (1999).

${ }^{13}$ K. L. Ngai and C. T. Moynihan, Bull. Mater. Res. Soc. 23, 51 (1998).

${ }^{14}$ K. L. Ngai and C. León, Phys. Rev. B 60, 9396 (1999).

${ }^{15}$ K. L. Ngai, R. W. Rendell, and H. Jain, Phys. Rev. B 30, 2133 (1984).

${ }^{16}$ C. León, M. L. Lucia, J. Santamaria, M. A. Paris, J. Sanz, and A. Várez, Phys. Rev. B 54, 184 (1996).

${ }^{17}$ C. León, J. Santamaria, M. A. Paris, J. Sanz, J. Ibarra, and L. M. Torres, Phys. Rev. B 56, 5302 (1997).

${ }^{18}$ A. Pimenov, J. Ullrich, P. Lunkenheimer, A. Loidl, and C. H. Rüscher, Solid State Ionics 109, 111 (1998).

${ }^{19}$ C. León, M. L. Lucía, and J. Santamaría, Phys. Rev. B 55, 882 (1997).

${ }^{20}$ K. L. Ngai, Comments Solid State Phys. 9, 121 (1979); 9, 141 (1980); K. L. Ngai and K. Y. Tsang, Phys. Rev. E 60, 4511 (1999).

${ }^{21}$ J. E. Bauerle and J. Hrizo, J. Phys. Chem. Solids 30, 565 (1969). 